\title{
The role of actin in capacitation-related signaling: an in silico and in vitro study
}

\author{
Nicola Bernabò , Paolo Berardinelli, Annunziata Mauro, Valentina Russo, Pia Lucidi, Mauro Mattioli and \\ Barbara Barboni
}

\begin{abstract}
Background: The signalling cascades involved in many biological processes require the coordination of different subcellular districts. It is the case of the pathways involved in spermatozoa acquisition of fertilizing ability (the so called "capacitation"). In the present work the coordination of subcellular signalling, during the boar sperm capacitation, was studied by a computational and experimental approach. As first the biological network representing all the molecular interactions involved in capacitation was build and analyzed, then, an experimental set up was carried out to confirm the computational model-based prediction.
\end{abstract}

Results: The analysis of computational model pointed out that the "actin polymerization" node had some important and unique features:

- it is one of the most connected nodes,

- it links in a specific manner all the intracellular compartments,

- its removal from the network did not affect the global network topology but caused the loss of five important nodes (and among them the "plasma membrane" and "outer acrosome membrane" fusion).

Thus, it was suggested that actin polymerization could be involved in the signaling coordination of different subcellular districts, and that its functional ablation could compromise spermatozoa ability to complete the capacitation (while the main signaling pathway remained unaffected). The experiments, carried out inhibiting the actin polymerization in capacitating boar spermatozoa by the administration of cytocalasin D (CD), demonstrated that the CD treatment inhibited spermatozoa ability to reach the full fertilizing ability, while, the examined signaling pathways (membrane acquisition of chlortetracicline pattern C, protein tyrosine phosphorylation, phospholipase C- $\gamma 1$ relocalization, intracellular calcium response to zonae pellucidae) remained effective, thus, confirming the model-based hypothesis.

Conclusions: The model based-hypothesis was confirmed by the reported data obtained with the in vitro experiments, which strengthen the idea that the actin cytoskeleton is not only a mechanical support for the cell, but that it exerts a key role in signaling during the sperm capacitation.

\section{Background}

One of the most important events in the history of life was the eukaryotes evolution that started with the onset of intracellular compartmentalization, approximately 1.62.1 billion years ago [1]. This fundamental step had enormous consequences on cell organization and function. In fact, in one hand, the appearance of specialized districts

\footnotetext{
* Correspondence: nbernabo@unite.it

Department of Comparative Biomedical Sciences, University of Teramo, 64100 Teramo, Italy
}

within the cytoplasm increased the efficiency of metabolic and signaling pathways, in the other hand, cell complexity increased. In fact, the development of organelles determined the existence of specific subcellular microenvironments characterized by different biochemical properties. Thus, the cell complexity arose either by the large number of molecular components involved in signal transduction and by the reciprocal connections and spatial relationship existing among them [2]. In addition, the spatial segregation of molecules and chemical reactions
C Biomed Central

C 2011 Bernabò et al; licensee BioMed Central Ltd. This is an Open Access article distributed under the terms of the Creative Commons Attribution License (http://creativecommons.org/licenses/by/2.0), which permits unrestricted use, distribution, and reproduction in any medium, provided the original work is properly cited. 
allowed that the same molecule could be responsible of very different signals. "We already have signaling "wires" distinguished by the identity of the molecules in the pathways. Compartmentalization duplicates these existing wires and separates them in space. This multiplies the number of signals they can carry" [2]. This increasing complexity imposes new biological problems as the emergence of new proprieties of cellular systems and the need of the presence of a/some conductor/s which harmonize the function of subcellular compartments. Recently the interpretation of the molecular mechanisms involved in cell signaling is made possible by new computational approaches that allow to modellize cellular functions as a network of integrated and coordinated signals operating within subcellular districts interconnected to each other $[3,4]$. One clear example is represented by sperm cells, whose biochemical mechanisms leading to capacitation during the post-ejaculatory life, were recently described by using the biological networks formalism [5]. The spermatozoa offer several advantages to adopt this kind of approach since:

- the main molecular events occurring during sperm capacitation are largely studied for biological and clinical reasons;

- their maturation can be experimentally reproduced under in vitro condition and assessed by measuring their ability to undergo acrosomal reaction (AR) after zonae pellucidae stimulation (ZP) or to in vitro fertilize the oocytes (IVF);

- their molecular composition is stable since the nucleus of the male germ cells is transcriptionally silent except for the mitochondrial protein translation of nuclear-encoded proteins [6].

Starting from these premises, the present work has been carried out, first, to study, using a computational approach, the mechanisms involved in the coordination of the molecular events that take place in the different subcellular districts during the post-ejaculatory spermatozoa maturation, the capacitation. Then, an experimental set-up was developed to confirm the model-based prediction. To this aim the events potentially acting as coordinators were inhibited by specific drugs during the spermatozoa incubation under capacitating conditions. The effects of this inhibition were assessed on functional spermatozoa status (ZP-induced AR), and on the major cellular events involved in the acquisition of full fertilizing ability (membrane acquisition of chlortetracycline pattern $\mathrm{C}$, protein tyrosine phosphorylation, phospholipase $C-\gamma 1$ relocalization, intracellular calcium response to $\mathrm{ZP})$.

\section{Results}

Computational model of spermatozoa capacitation

The main topological parameters of the network representing the capacitation are shown in Table 1. The distribution of node linkages followed a power law, represented by the generic equation:

$$
y=a x^{-b}
$$

were

$$
\mathrm{a}=70.915
$$

and

$$
\mathrm{b}=-1.578
$$

The $r, R^{2}$ coefficients were, respectively, 0.825 and 0.849 .

The clustering coefficient distribution does not follow a power law, thus, the results of power law fitting of clustering coefficient distribution were: $r=0.120 ; R^{2}=0.232$.

The most connected nodes are showed in Table 2.

The analysis of the network topology revealed that only tree nodes bound all the subcellular compartments involved in post-ejaculatory signalling: $\left[\mathrm{Ca}^{2+}\right]_{\mathrm{i}}$, ATP, and "actin polymerization". $\left[\mathrm{Ca}^{2+}\right]_{i}$ and ATP are the most linked nodes and bound about one third of the nodes $(48 / 151)$. More interesting is the behaviour of the "actin polymerization" node, represented in Figure 1, that links a smaller number of nodes (8) located in all the subcellular compartments (membrane, cytosol, cytoskeleton, mitochondria, acrosome).

The removal of the "actin polymerization" node from the capacitation network had modest consequences on the network topological parameter (as shown in Table 3), but caused the loss of 5 nodes: OAM fusion, PM fusion,

Table 1 Main topological parameters of capacitation network

\begin{tabular}{lc}
\hline Parameter & Value \\
\hline$N^{\circ}$ nodes & 153 \\
$N^{\circ}$ edges & 204 \\
Clustering coefficient & 0.056 \\
Diameter & 12 \\
Averaged no neighbours & 2.654 \\
Char. path length & 4.995 \\
\hline
\end{tabular}

The number of nodes represent the total number of molecules involved, the number of edges represents the total number of interaction found, the clustering coefficient is calculated as $C l=2 n l / k(k-1)$, where $n l$ is the number of links connecting the $k \mathrm{l}$ neighbours of node $I$ to each other, the network diameter is the largest distance between two nodes, the Averaged $n^{\circ}$ neighbours represent the mean number of connection of each node, the Char. path length gives the expected distance between two connected nodes. 


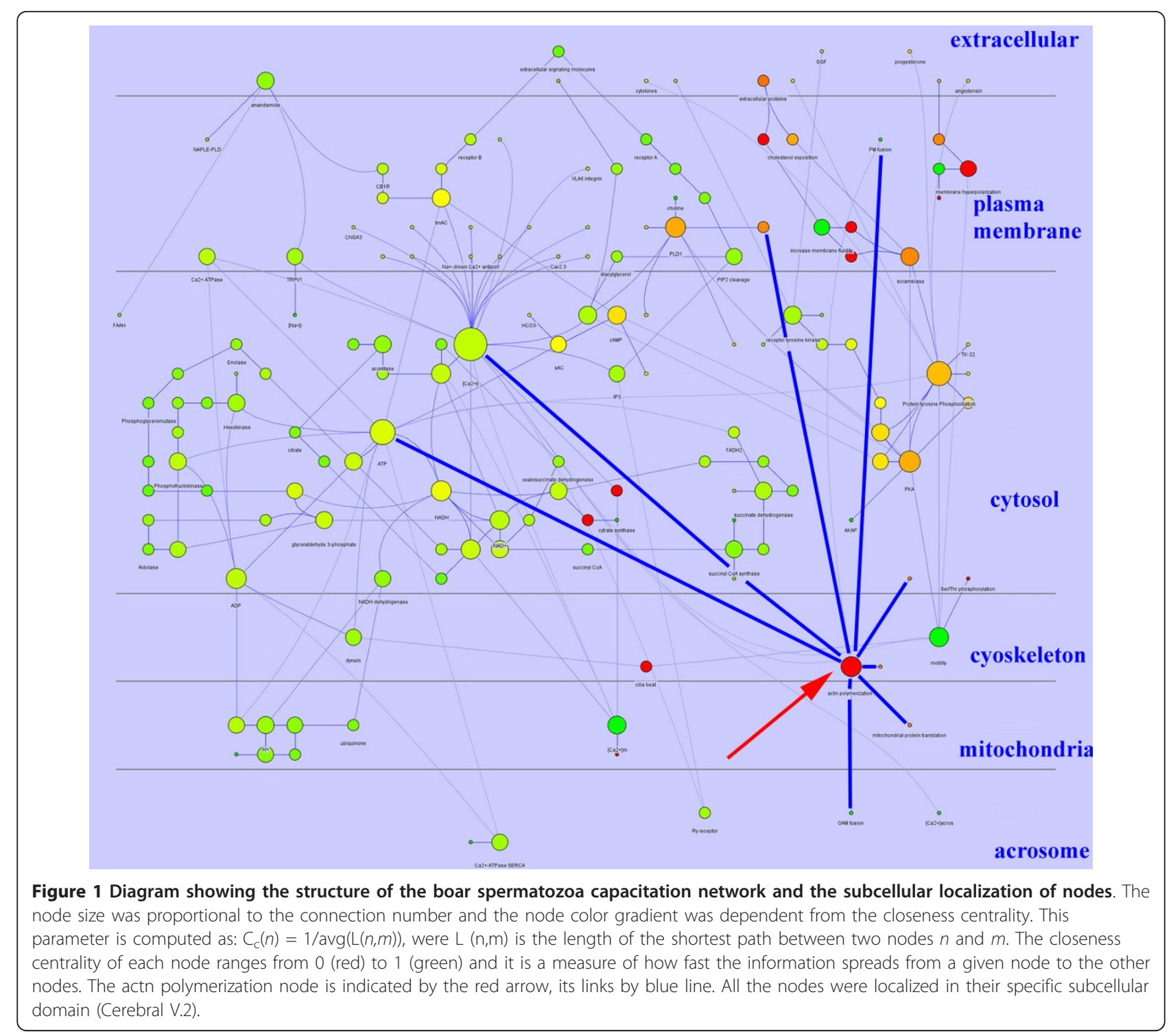

Table 2 Most connected nodes (the hubs) of capacitation network

\begin{tabular}{cc}
\hline Node & Number of links \\
\hline$\left[\mathrm{Ca}^{2+}\right]_{\mathrm{i}}$ & 28 \\
ATP & 15 \\
Tyr phosphorylation & 13 \\
PKA & 9 \\
ADP & 8 \\
PLD1 & 8 \\
NADH & 8 \\
Actin polymerization & 8 \\
\hline
\end{tabular}

Table 3 Main topological parameters of capacitation network after "actin polymerization" node removal

\begin{tabular}{lc}
\hline Parameter & Value \\
\hline $\mathrm{N}^{\circ}$ nodes & 152 \\
$\mathrm{~N}^{\circ}$ edges & 196 \\
Clustering coefficient & 0.056 \\
Diameter & 12 \\
Averaged $\mathrm{n}^{\circ}$ neighbours & 2.566 \\
Char. path length & 6.071 \\
Degree distribution & $\mathrm{b}=-1.563$ \\
& $\mathrm{r}=0.809 \mathrm{R}^{2}=0.898$
\end{tabular}

The number of nodes represent the total number of molecules involved, the number of edges represents the total number of interaction found, the clustering coefficient is calculated as $C l=2 n l / k(k-1)$, where $n l$ is the number of links connecting the $k \mid$ neighbours of node I to each other, the network diameter is the largest distance between two nodes, the Averaged $n^{\circ}$ neighbours represent the mean number of connection of each node, the Char. path length gives the expected distance between two connected nodes. 
G-actin, F-actin, mitochondrial protein translation (see Figure 2).

\section{Phalloidin staining}

The distribution of the fluorescence within the spermatozoa was assessed, allowing the identification of two different patterns:

- pattern A) low actin polymeriziation: the phalloidin fluorescence emission is low and is mainly localized over the midpiece and the post acrosomial region (Figure 3A);

- pattern B) high actin polymerization: the phalloidin fluorescence emission is high and localized over the midpiece and the whole sperm head (Figure 3B).

The majority of freshly ejaculated spermatozoa localized a faint fluorescence emission in the midpiece and in the post acrosomal region (pattern A in 99 of 108 analysed spermatozoa). After $4 \mathrm{~h}$ of incubation under control conditions, the majority of spermatozoa increased their content of Factin that resulted to be localized over sperm head (pattern $\mathrm{B}$ in 99 of 112 analysed spermatozoa; $\mathrm{p}<0.01$ vs. T0). The $\mathrm{CD}$ treated spermatozoa, displayed a fluorescence signal similar to that recorded at T0 (pattern A in 96 of 107 analysed spermatozoa; $\mathrm{p}>0.05$ vs. T0 and $\mathrm{p}<0.01$ vs. CTR).

\section{ZP-induced AR}

The percentage of acrosome reacted spermatozoa recorded during incubation in control condition ranged from about $5 \%$ at the beginning of incubation to about $10 \%$ after $4 \mathrm{~h}$. The sZP addition to the samples made possible to quantify the percentage of the spermatozoa responding to the physiological stimulus with the exocytosis of the acrosomal content (i.e. the cells which completed the capacitation process), which after the $4 \mathrm{~h}$

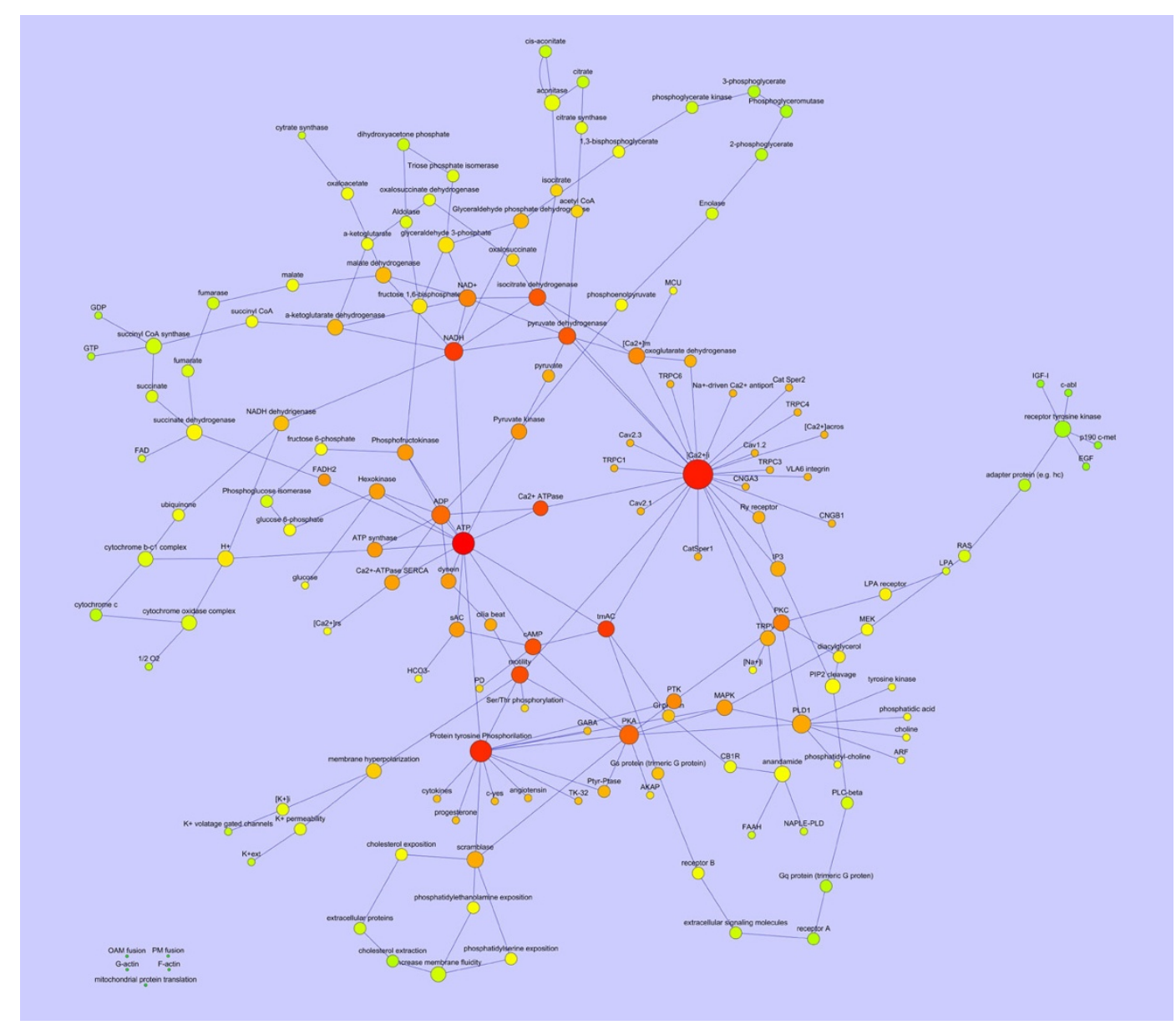

Figure 2 Diagram showing the structure of the boar spermatozoa capacitation network after "actin polymerization" node removal The node size was proportional to the connection number and the node color gradient was dependent from the closeness centrality. This parameter is computed as: $C_{c}(n)=1 / \operatorname{avg}(L(n, m))$, were $L(n, m)$ is the length of the shortest path between two nodes $n$ and $m$. The closeness centrality of each node ranges from 0 (red) to 1 (green) and it is a measure of how fast the information spreads from a given node to the other nodes. The spatial network arrangement was obtained by using the Cytoscape Spring-embedded Layout (see the text for explanation). 

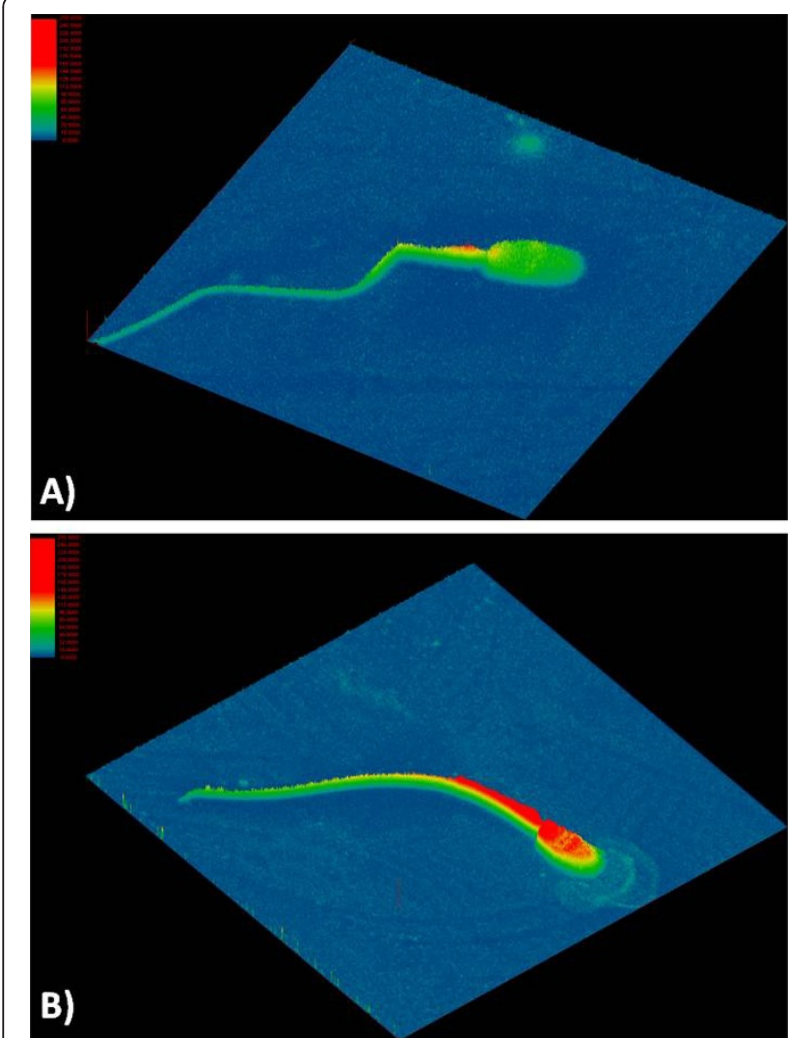

Figure 3 Confocal image representing actin localization in ejaculated and in capacitated spermatozoa. Confocal image representative of TRITC-phalloidin distribution on freshly ejaculated spermatozoon showing a faint fluorescence emission over the midpiece and post-acrosomal region (pattern A; panel A) and of incubated spermatozoon, displaying high fluorescence emission over the whole head (pattern B; panel B).

incubation reached about the 30\% (Figure 4A). The CD treated spermatozoa showed an incidence of spontaneous AR similar to that recorded in the control samples during all the $4 \mathrm{~h}$ of incubation ( $\mathrm{p}>0.05)$, instead, the sZP addition promoted the AR in a markedly reduced percentage of cells ( $p<0.01$ vs. CTR), see Figure 4B.

\section{Chlortetracycline staining}

The spermatozoa incubated under control conditions, at the beginning of incubation, showed three different patterns of chlortetracycline (CTC) stain in agreement with Mattioli et al. [7]:

- about $80 \%$ of spermatozoa displayed a faint fluorescence uniformly distributed over the head (pattern A); - about the $10 \%$ spermatozoa displayed the fluorescence concentrated in the post-acrosomal region (pattern B);

- about the $10 \%$ displayed the fluorescence concentrated in the acrosome (pattern $\mathrm{C}$ ): these

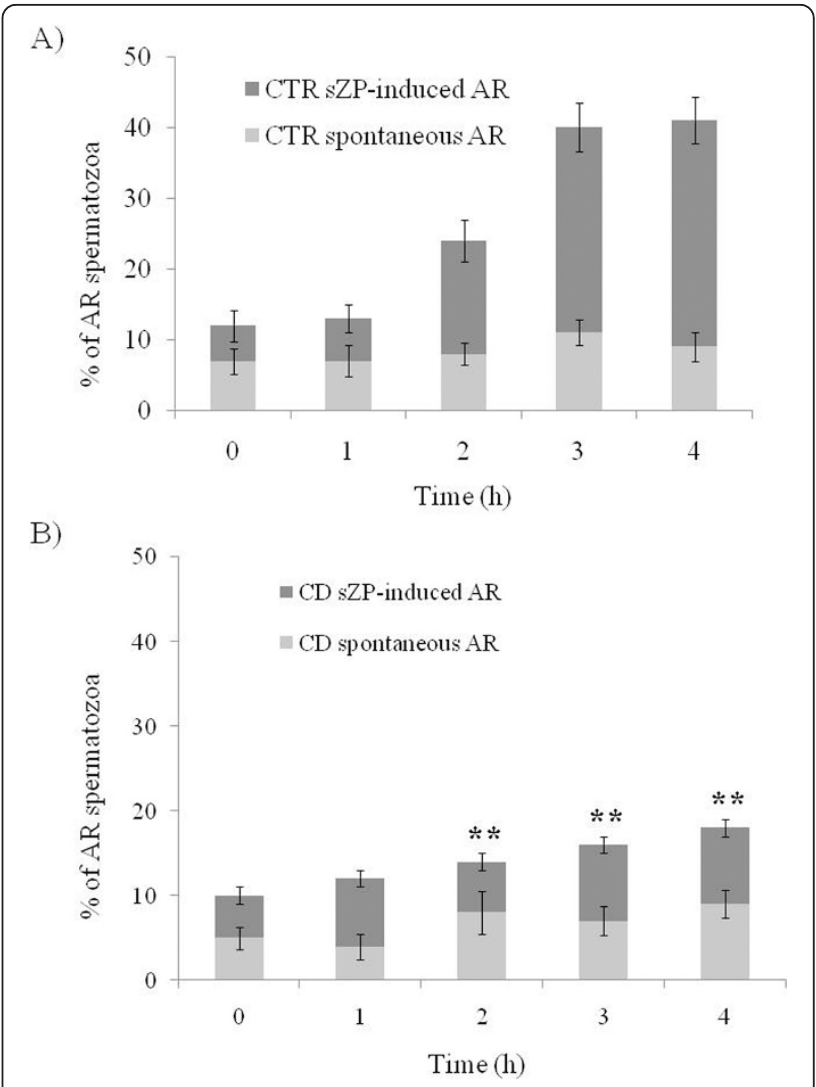

Figure 4 Histogram representing the percentage of spermatozoa undergoing spontaneous and SZP-induced AR in CTR (A) and CD (B) treated spermatozoa. Histograms showing the percentage of spermatozoa undergoing spontaneous (light gray) or sZP-induced (dark gray) AR in spermatozoa incubated under control conditions (panel A) or in the presence of $C D$ (panel B). All the values are represented as mean $\pm S D$. ${ }^{*}=p<0.01$ vs. CTR.

spermatozoa, in keeping with Mattioli et al. [7], were considered to have completed the capacitation-related membrane reorganization.

After $4 \mathrm{~h}$ of incubation the percentage of the three patterns was markedly different: pattern A decreased to about $40 \%$, pattern $B$ remained at about $10 \%$, while, pattern $\mathrm{C}$ reached $50 \%$. As evident in Figure 5, the treatment with $C D$ did not affect significantly the percentage of spermatozoa showing the different CTC stain patterns.

\section{Tyrosine phosphorylation}

The tyrosine phosphorilation pattern of freshly ejaculated male gametes (T0), or of spermatozoa incubated under control condition (CTR), or in the presence of CD was assessed. As summarized in Figure 6, after the incubation the tyrosine phosphorylation pattern changed regardless of the cultural condition adopted (CTR or CD). 


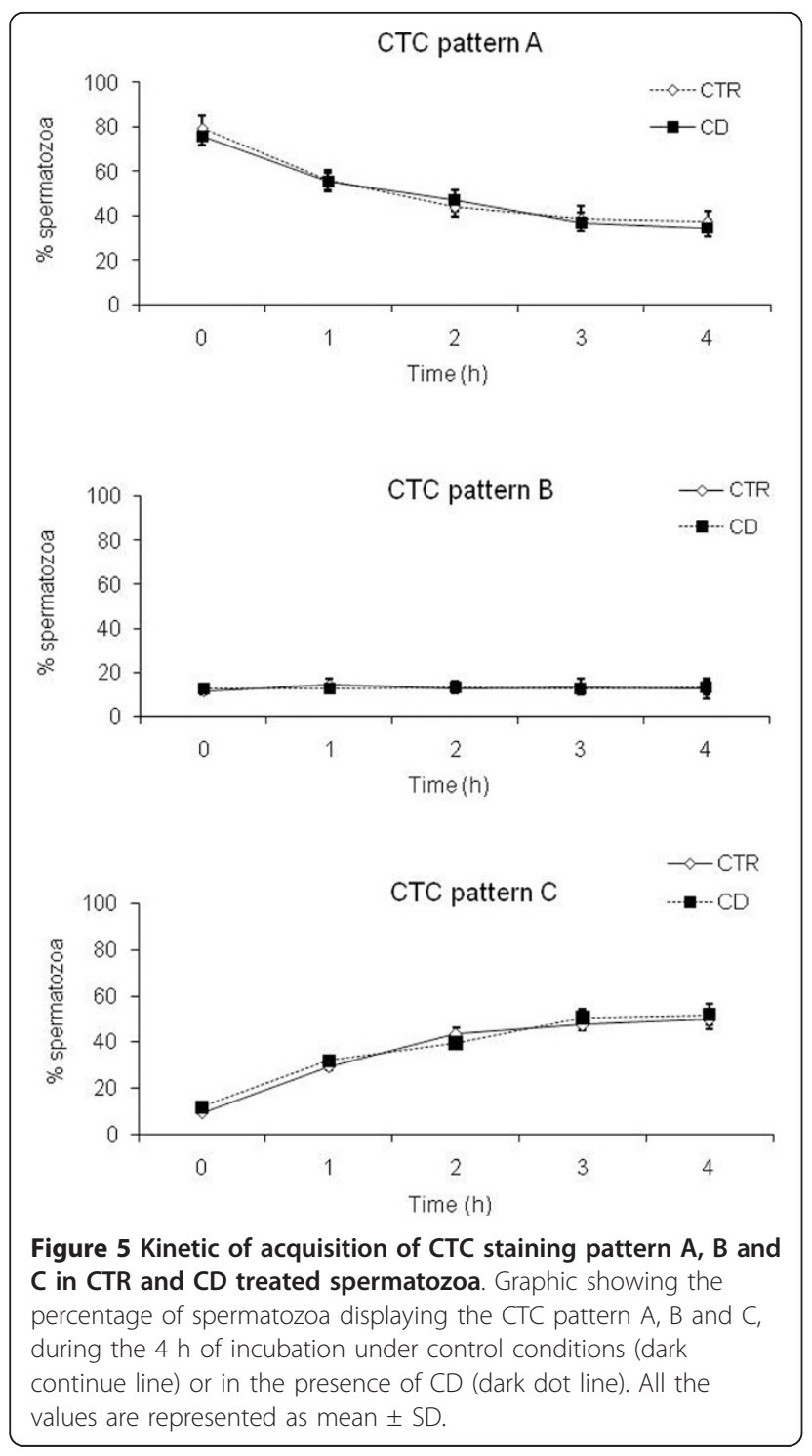

\section{Phospholipase C- $\gamma 1$ relocalization}

Phospholipase C- $\gamma 1$ (PLC- $\gamma 1$ ) protein expression studied with Western Blotting revealed that this protein modified its localization during the interval of culture. In fact, as shown in Figure 7, uncapacitated sperm cells (T0) strongly expressed PLC- $\gamma 1$ within the cytoplasm, while, the incubation caused its translocation into the membrane compartment both in CTR and in CD samples.

Ultrastructural analysis performed under preembedding condition, confirmed the membrane traslocation of PLC- $\gamma 1$ in incubated spermatozoa maintained either in the presence or absence of $\mathrm{CD}$, confirming the Western Blot analysis. In fact, uncapacitated spermatozoa displayed very few gold particles, indicative of PLC$\gamma 1$, on their membranes (Figure 8A), while, in in vitro incubated cells, independently from the used treatment,

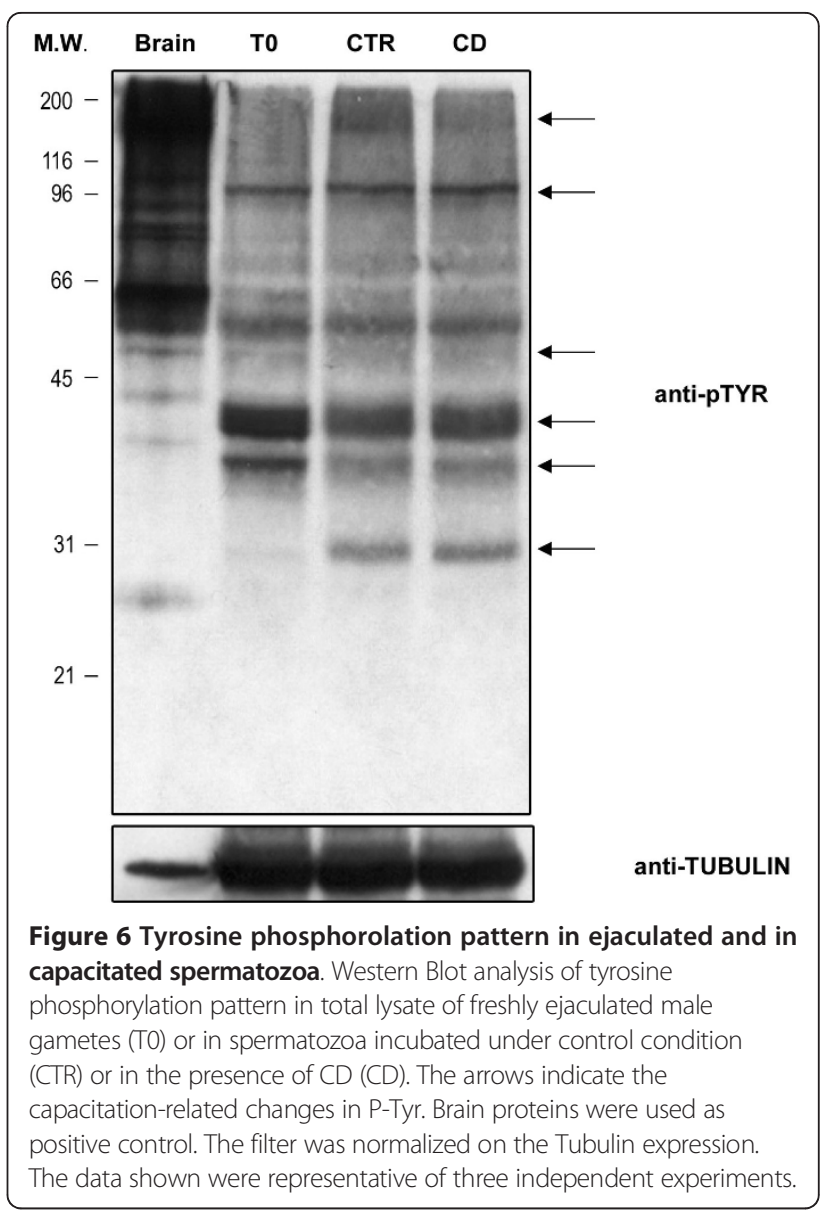

the number of gold particles localizing the protein on the membrane notably grew (Figure 8B).

\section{ZP-induced intracellular calcium concentration rise}

The coincubation with the SZP in spermatozoa capacitated under control conditions caused an evident $\left[\mathrm{Ca}^{2+}\right]_{\mathrm{i}}$

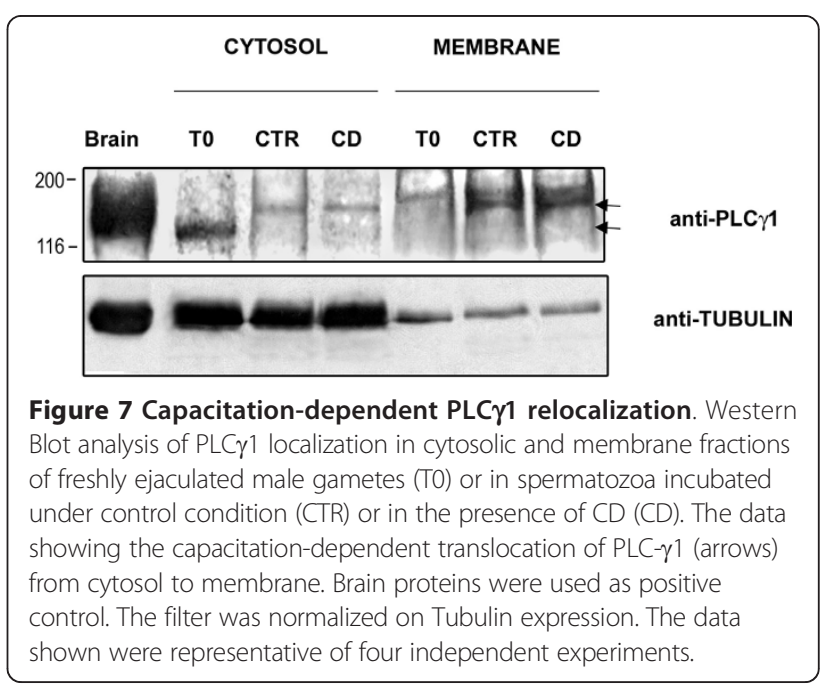




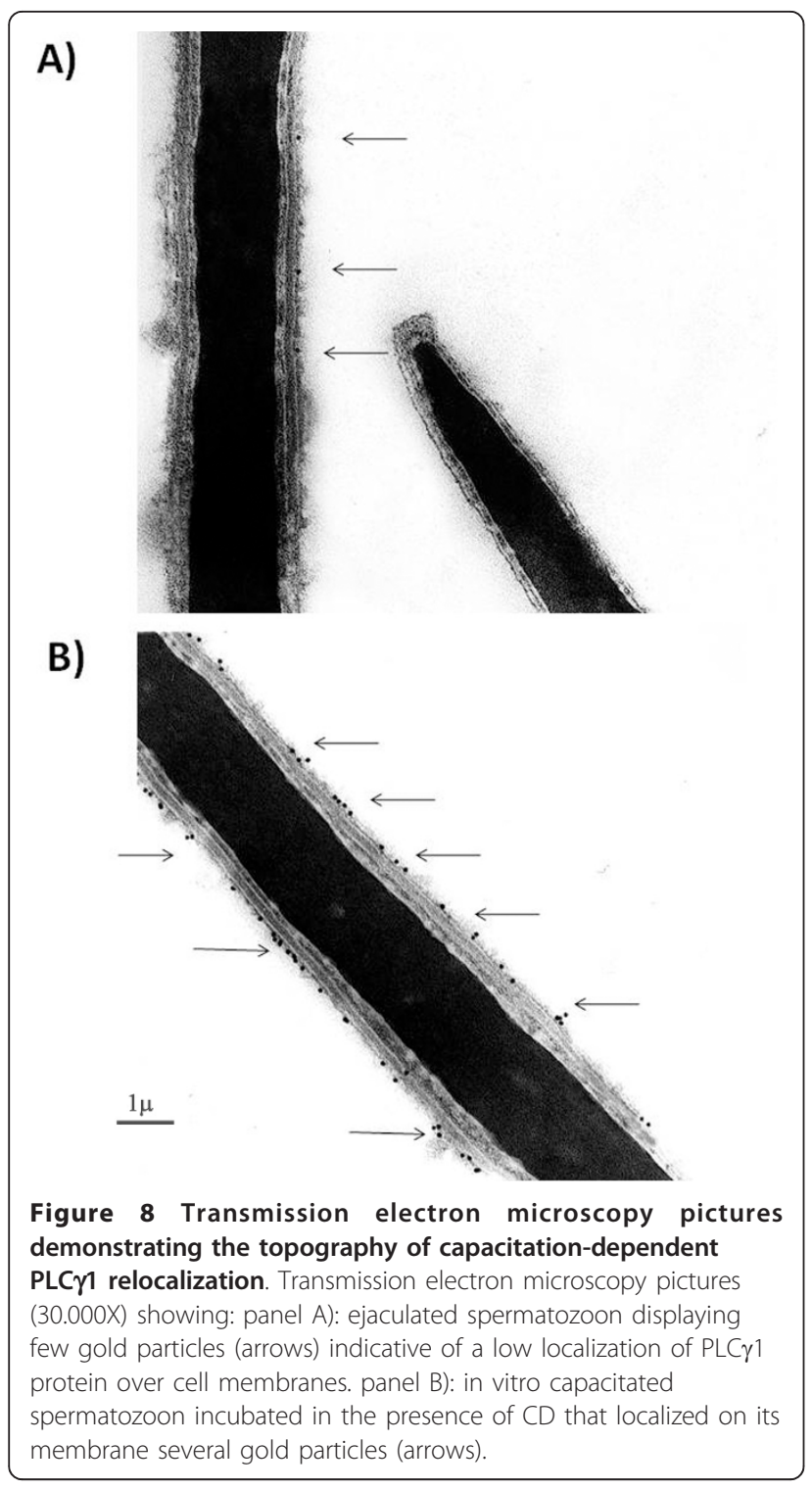

elevation in the $36 \%$ of sperm cells (27 of 75 examined cells): the intracellular $\mathrm{Ca}^{2+}$ peaked in 1-2 sec and lasted 20-40 sec before $\left[\mathrm{Ca}^{2+}\right]_{\mathrm{i}}$ returning at a basal level. In the presence of $C D$ the ability and the kinetic to respond with a $\left[\mathrm{Ca}^{2+}\right]_{\mathrm{i}}$ rise to the sZP coincubation (Figure 9) was maintained in the $38 \%$ (32 of 83 examined cells) of spermatozoa ( $\mathrm{p}>0.05$ vs. CTR).

\section{Discussion}

Aim of this work was to study the molecular event/s involved in the coordination of the biochemistry of the subcellular compartments during spermatozoa acquisition of fertilizing ability (the so called capacitation) using a predictive computational modelisation and the in vitro experimental approach as a confirmation. Thanks the in silico experiment the node acting as a coordinator for the signaling cascades of different subcellular districts was identified. Then, the consequences on cellular function of its inhibition were experimentally evaluated in vitro. This goal was targeted by representing the biochemical machinery that drives the capacitation as a biological network that, on the basis of its topological proprieties, was considered as a scale free network. In fact, the node degree (or connectivity), $k$, which indicates how many links the node has to other nodes, followed a power-law distribution of the number of links per node and, in parallel, the tendency to develop clusters of nodes, the so called clustering coefficient CI [CI $=2 n \mathrm{I} / k(k-1)$, where $n \mathrm{I}$ is the number of links connecting the $k I$ neighbors of node I to each other] was independent of the number of links per node. This architecture conferred to the capacitation network high signaling efficiency and robustness against random failure [5] and, in keeping with the Barabási-Albert model [8], made evident that a relatively small number of nodes (the hubs) were highly connected and most of the nodes were scarcely linked. Thus, it was possible to identify the hubs, i.e. the molecules playing a key role in post-ejaculatory maturation of spermatozoa and to localize them in different subcellular districts. In addition, the functional meaning and the localization of all the molecules involved in capacitation were studied and the nodes that interconnect the intracellular compartments were identified. It is really impressive that these nodes were only three: $\left[\mathrm{Ca}^{2+}\right]_{\mathrm{i}}$, ATP and "actin polymerization". More in detail $\left[\mathrm{Ca}^{2+}\right]_{i}$ and ATP are linked with a high number of nodes (28 and 15 respectively) and exerted the function of ubiquitous second messengers $\left(\left[\mathrm{Ca}^{2+}\right]_{\mathrm{i}}\right)$ or of metabolic sustain (ATP). It is important to note that it has been recently demonstrated that their ablation from the network causes the collapse of network structures [5]. On the contrary, the "actin polymerization" bounds in a specific way, having only 8 links, all the subcellular compartments involved in capacitation (cytosol, cytoskeleton, mitochondria and acrosome). It is suggestive that it does not link the nucleus, the only compartment that remains stable until fertilization.

In particular it links:

- phosphatidic acid (i.e. the product of PLD activity): it is known that actin polymerization depends on PLD activation, which occurs via the HCO3 2/ cAMP/PKA pathway or via the G-protein coupled receptor (GPCR) (LPA-receptor)/PKC pathway [9];

- ATP: the actin is an ATPase and its polymerization depends on ATP cellular concentration, in addition, the ATP is the most important molecule of spermatozoa energy metabolism. In fact, the metabolic energy production in spermatozoa depends on glycolysis and/or on mitochondrial oxidative [10]; 

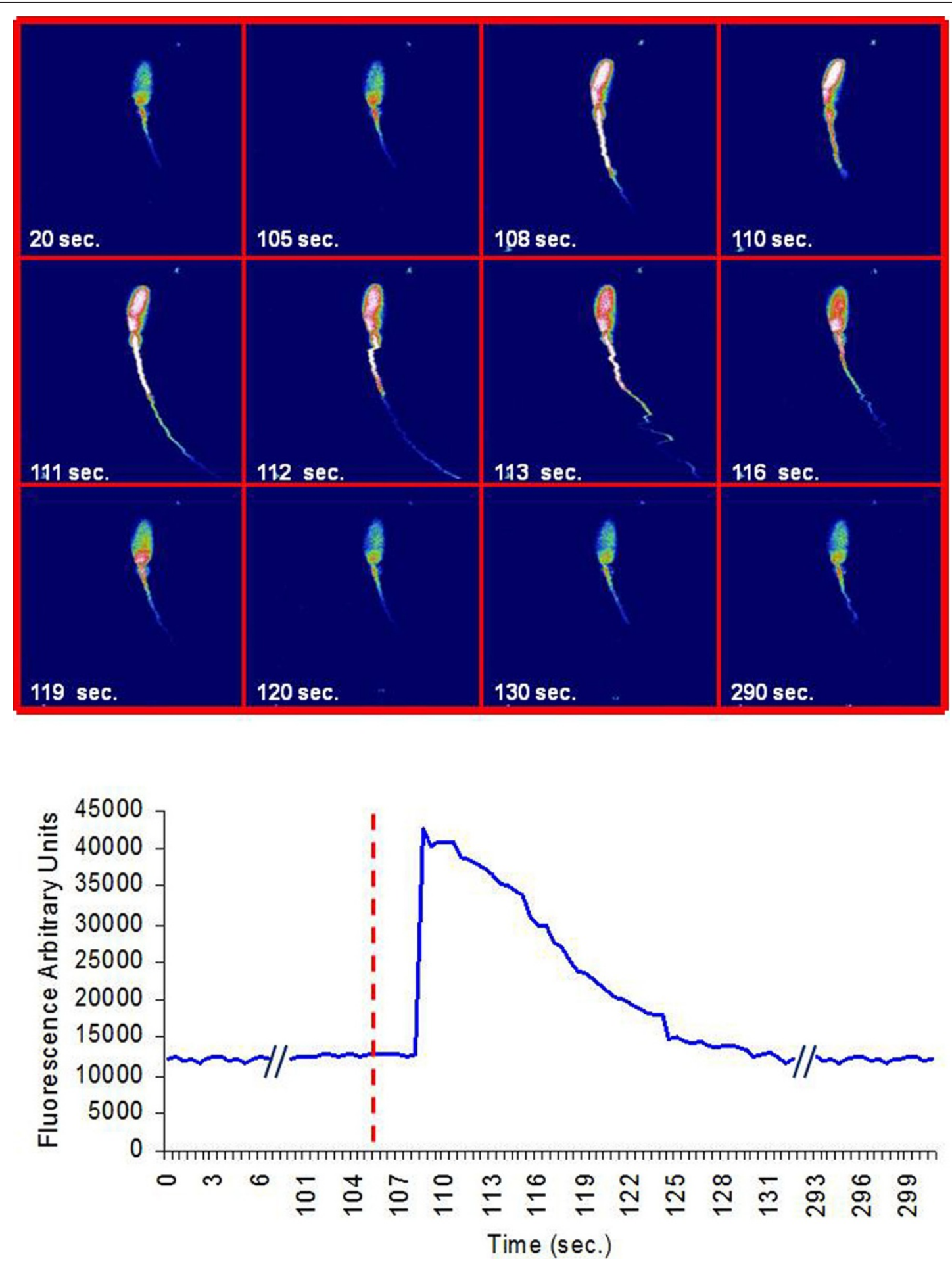

Figure 9 An example of confocal image gallery of CD treated spermatozoa loaded with Fluo-3AM exposed to sZP. An example of confocal image gallery of CD treated spermatozoa loaded with Fluo-3AM exposed to sZP (vertical red line). Notice that the rise in the $\left[\mathrm{Ca}^{2+}\right]_{i}$ in spermatozoon is evident after $<10 \mathrm{sec}$. from sZP addition, whereas return on the baseline occurs after about $30 \mathrm{sec}$. 
- F-actin and G-actin: for recent reviews on actin polymerization see [11-13];

- $\left[\mathrm{Ca}^{2+}\right]_{\mathrm{i}}$ : the intracellular calcium is one of the most important second messengers that drives the intracellular signaling during capacitation;

- OAM and PM fusion: see below for explanation;

- Protein synthesis: the incubation with the mitochondrial translation inhibitor D-chloramphenicol almost completely blocked spermatozoa actin polymerization [6].

In this context it is important to note that the links of actin polymerization involve three of the most linked nodes found in capacitation (ATP, $\left[\mathrm{Ca}^{2+}\right]_{\mathrm{i}}$ and PLD) and that this node was per se one of the most linked node. Thus, it is possible to hypothesize that a message involving the actin polymerization node could reach all the cell districts, perturbing the whole signal transduction system of spermatozoa.

In addition, it has been found that the removal of "actin polymerization" node caused an important effect: the main topological parameters of the capacitation network were virtually unaffected but some nodes remained out from the network: OAM fusion, PM fusion, G-actin, F-actin, mitochondrial protein translation. This finding allows to predict that the functional ablation of actin polymerization during the in vitro capacitation could cause the spermatozoa inability to undergo AR (the OAM and PM fusion did not take place) while, the different signalling pathways remained unaffected.

To obtain an experimental confirmation of the modelbased prediction, the actin polymerization was inhibited, as clearly confirmed by confocal analyses, by the incubation of spermatozoa under capacitating condition in the presence of CD.

The effect of this drug on the whole cell function was immediately evident: the treated spermatozoa lost the ability to undergo AR when exposed to the physiological agonist, the sZP. In parallel, the biochemical analyses showed that the main signaling pathways involved in capacitation were unaffected by the treatment. Particularly the capacitation implies a marked calcium-dependent rearrangement of membrane structure that, in turn, determines the increase of the plasma membrane (PM) and of the outer acrosome membrane (OAM) fusogenicity. This event resulted to be unaffected by $\mathrm{CD}$ administration as demonstrated by CTC staining.

Another important signaling pathway involves the protein tyrosine phosphorylation, via $\mathrm{sAC/CAMP/PKA}$. It is known that the protein tyrosine phosphorylation pattern of spermatozoa changes during the capacitation in several species such as human, mice, cattle, pig, hamster and cat [14-16]. In boar spermatozoa a typical protein phosphorylation pattern has been identified [17], and the CD treatment did not exert any detectable effect on this parameter.

The PLC- $\gamma 1$ plays a key role in coupling actin cytoskeleton and membrane dynamics to the calcium metabolism. When activated this enzyme migrates from the cytosol to the membrane [18] where it hydrolyses the phosphatidylinositol 4,5-bisphosphate [PI(4,5)P2] generating inositol 1,4,5-trisphosphate, a universal calciummobilizing second messenger, and diacylglycerol, an activator of protein kinase $C$. the present results showed that the PLC- $\gamma 1$ translocation was unaffected by the presence of $C D$ in the capacitation medium.

Ultimately, the effect of spermatozoa incubation under capacitating condition in presence of $C D$ on calcium metabolism was tested. In fact, the spermatozoa signaling machinery strictly depends on intracellular calcium concentration [19-21]. During the capacitation the $\mathrm{Ca}^{2+}$ behaves as a second messenger converting extracelluar stimuli in chemical response involving a myriad of molecular system, such as, protein kinase $\mathrm{C}$ (PKC), protein kynase $C$ (PKA), actin, and many others. The completion of capacitation enables the spermatozoa to respond to the interaction with oocyte zona pellucida with a very fast surge of $\left[\mathrm{Ca}^{2+}\right]_{\mathrm{I}}$, during AR. In the present work the incubation of spermatozoa in presence of $\mathrm{CD}$ did not exert any detectable effect on this event.

From these findings it is evident that the spermatozoa inability to fulfill the physiological goal, the ZP-induced $A R$, is paralleled by the maintained efficiency of the main signaling pathways, in agreement with the computational model-based prediction.

The data until now available attribute to the actin dynamics a structural role during capacitation and AR. In particular, it is known that gradually the PM acquires the ability to fuse with the OAM thanks to the remodelling of its lipid composition and architecture [9,19,22-24]. The developing network of actin acts as a diaphragm between the two membranes avoiding their fusion. Once the capacitation was completely achieved and the physiological stimulus (ZP proteins) was detected, the AR takes place and the ZP-induced calcium peak causes the fast depolymerisation of actin structure. If this model was correct it was reasonably to expect that the block of actin polymerization (i.e. the treatment of spermatozoa with $\mathrm{CD}$ ) in early phases of capacitation leads the increase in the percentage of spermatozoa showing the loss of acrosome integrity due to the inefficiency of separation of PM and OAM. In our experiments, on the contrary, it was found that, even in the absence of the actin network, the PM and OAM did not fuse. Thus, the results of the present work could complete and revamp the current concept, leading us to hypothesize that in swine spermatozoa, as demonstrated in other cells, the role of actin cytoskeleton overcomes this merely structural function. 
It is possible to conceive that, linking all the cellular districts involved in capacitation, the actin polymerization could have a role of general coordination of this pivotal biological event. In particular, it is noteworthy that this node links three hubs of the system, thus, strengthening the hypothesis that actin polymerization acts as an important node of coordination trough the information flow. This supposition is in keeping with the newly emerging evidence that in different cellular systems the cytoskeleton is not only a mechanical support for the cell, but exerts a key role in signaling. In fact, it was proposed that "independent of its mechanical strength, the filaments of the cytoskeleton form a continuous, dynamic connection between nearly all cellular structures, and they present an enormous surface area on which proteins and other cytoplasmic components can dock" [25]. This is strengthened by the finding that the plasma membrane surface area of a $20-\mu \mathrm{m}$-diameter generic cell is on the order of $700 \mu \mathrm{m}^{2}$, in contrast, the total surface area of a typical concentration of $10 \mathrm{mg} / \mathrm{ml} \mathrm{F}$-actin is $47,000 \mu \mathrm{m}^{2}$ [25] and that the diffusion along cytoskeletal tracks could be a reliable alternative to other established ways of intracellular trafficking and signaling, and could therefore provide an additional level of cell function regulation [26]. One implication of this role is that, together with other well known molecules involved in intracellular signaling, such as $\mathrm{Ca}^{2+}$ or ATP, the actin cytoskeleton might provide a signal transduction route and macromolecular scaffold, which, during eukaryotic evolution, contributes to the spatial organization of signaling pathways components [27].

\section{Conclusions}

From these data it is possible to take some considerations:

1. it is possible to speculate that the male germ cell representation as a biological network could be an important tool, because it would allow to study not only the molecular components of these cells, but also their intracellular localization and intermolecular interactions in signaling pathways. In this context it is important to note that in complex systems the whole system is more than the sum of its single components; 2 . the present findings could be discussed as the evidence that spermatozoa behave as complex systems. In this optic the acquisition of the fertilizing ability is a system propriety that emerges when these cells are considered in their whole signaling network. If the single subcellular element is unperturbed by an external factor, but the coordination among all of them is broken, the male gamete becomes unable to express the ability to physiologically respond to the stimulus (the sZP). In addition, the molecule/s acting as controller of cross-talking among the different intracellular compartments could become a sort of "Achilles' heel" of cellular signaling machinery, when compromised.

3. it was already known that the actin cytoskeleton is a highly dynamic structure involved in capacitation. From the present data it emerges that actin polymerization could be involved in coordination of signaling during the capacitation.

In our opinion these findings strength the usefulness of the combined use of a computational and an experimental approach, could have important implications in diagnostics and therapeutics of male infertility, as well in contraceptive strategies, and could contribute to the knowledge of the role played by cytoskeleton in cell signal transduction.

\section{Methods \\ Chemicals}

Chemicals were of the purest analytical grade. Fetal Calf Serum (FCS) was purchased from GIBCO BRL (Milano, ITALY). Fluo-3-AM, Pluronic F-127, propidium iodide (PI), Hoecst 33258 were from Calbiochem, (San Diego, $\mathrm{CA}$ ); Percoll, Pisum sativum agglutinin-FITC (fluorescein isothiocyanate) conjugated (FITC-PSA), Dulbecco with $\mathrm{Ca}^{++}$and $\mathrm{Mg}^{++}$, TCM199, bovine serum albumine (BSA), sodium pyruvate, glucose, calcium lactate and all others chemicals were from Sigma Chemical Co. (St. Louis, MO).

\section{Capacitation computational model}

The biological network representing the molecular events occurring during the capacitation of boar spermatozoa was realized in accordance with Bernabò et al. [5], introducing some specie-specific modification, using Cytoscape 2.6.3 software (http://www.cytoscape.org). The representation of subcellular compartments was made with the Cytoscape plugin Cerebral v.2 (http:// www.pathogenomics.ca/cerebral/).

To evaluate the effect of the removal of the "actin polymerization" node from the capacitation network a new network was realized omitting this node ("no actin polymerization network"). This network was spatially represented using the Cytoscape Spring-embedded Layout. This program is based on a force-directed paradigm. Network nodes are treated like physical objects that repel each other, such as electrons. The connections between nodes are treated like metal springs attached to the pair of nodes. These springs repel or attract their end points according to a force function. The layout algorithm sets the positions of the nodes in a way that minimizes the sum of forces in the network" [15].

The statistical and topological analyses of the networks were carried out, considering the network as 
undirected, by the Cytoscape plugin Network Analyzer (http://med.bioinf.mpi-inf.mpg.de/netanalyzer/).

\section{Spermatozoa preparation}

Semen samples were collected and processed by an already validated protocol $[28,29]$. The incubation under capacitating condition was carried out in TCM199 medium added with $13.9 \mathrm{mM}$ glucose, $1.25 \mathrm{mM}$ sodium pyruvate, $2.25 \mathrm{mM}$ calcium lactate and $100 \mu \mathrm{g} / \mathrm{ml}$ kanamycin $(300 \mathrm{mOsm} / \mathrm{kg}, \mathrm{pH}$ 7.4) at a final concentration of $1 \times 10^{8}$ cells $/ \mathrm{ml}$ for $4 \mathrm{~h}$ at $38.5^{\circ} \mathrm{C}$ in $5 \% \mathrm{CO}_{2}$ humidified atmosphere (Heraeus, Hera Cell) for $4 \mathrm{~h}$. Only the samples maintaining a mean viability, assessed as previously described (Barboni et al. 1995), of at least $80 \%$ at the end of the culture were considered for the following analysis. The sperm samples were processed as freshly ejaculated (T0), incubated under control conditions (CTR) or were constantly maintained in the presence of $20 \mu \mathrm{M}$ of citochalasin $\mathrm{D}(\mathrm{CD})$.

In addition, in order to verify the effect of $\mathrm{CD}$ treatment on the rate of actin polymerizaton the sperm samples (T0, CTR and CD) were stained, as described by Bernabò et al. [30], with TRITC-conjugated phalloidin, a molecule exhibiting an high binding affinity for F-actin. For each treatment, at least 100 spermatozoa were observed by using the confocal microscope (Radiance 2000, BioRad, UK).

\section{Detection of ZP-induced AR}

The competence of in vitro incubated spermatozoa to undergo AR in response to solubilised zonae pellucidae (ZP) co-incubation was further evaluated as a functional endpoint of the capacitative state. To this aim aliquots of spermatozoa were incubated under different experimental conditions, and after the incubation period were exposed for $30 \mathrm{~min}$ to solubilised $\mathrm{ZP}$ (sZP) $(10 \mathrm{ZP} / \mu \mathrm{l})$, obtained as reported $[29,31]$. The percentage of sZPinduced AR was detected by FITC-PSA and Hoechst staining [29,31]. Since spermatozoa incubated for in vitro capacitation spontaneously and variably exocytate their acrosomal content in the absence of any specific stimulus, the acrosomal exocytosis before exposing spermatozoa to ZP was considered as "spontaneous AR". This spontaneous $A R$ was subtracted from total $A R$, in order to obtain the incidence of the true sZP-induced AR, in keeping with Bernabò et al. [30].

\section{CTC staining}

The chlortetracycline stain (CTC) was used to evaluate the completion of calcium-dependent membrane remodelling, as previously described (Mattioli et al., 1996; Barboni, 1994). In detail, $10 \mu \mathrm{l}$ for each sperm sample incubated under different conditions (CTR or $\mathrm{CD}$ ) were stained, on a warm stage, with $10 \mu \mathrm{l} \mathrm{CTC} 750 \mu \mathrm{M} / \mathrm{L}$ (solubilized in TRIS- $\mathrm{HCl} 20 \mathrm{mM} ; \mathrm{NaCl} 130 \mathrm{mM}$; L-cisteina $5 \mathrm{mM}, \mathrm{pH}$ 7.8); after $30 \mathrm{sec} 10 \mu \mathrm{l}$ glutaraldehyde $1 \%$ and $10 \mu \mathrm{l}$ mounting medium were added. For each sample were assessed at least 200 spermatozoa and the percentage of spermatozoa displaying fluorescence pattern C indicative of capacitation (CTC fluorescence over the post acrosomal area) was calculated.

\section{Biochemical evaluation of tyrosine phosphorilation pattern and PLC $\gamma$-1 relocalization}

Samples of spermatozoa were centrifuged $(500 \times \mathrm{g}$ for $3 \mathrm{~min}$.), washed in $1 \mathrm{ml}$ of Phosphate Buffer Saline (PBS) $\mathrm{pH} 7.5$ and then resuspended in following lysis buffer (Buffer-A): 50 mM Tris-HCL pH 7.5, $150 \mathrm{mM} \mathrm{NaCl}, 2$ mM EDTA pH 8, 1 mM Phenyl Methyl Mulphonyl Fluoride (PMSF), $1 \mathrm{mM}$ Sodium Orthovanadate $\left(\mathrm{Na}_{3} \mathrm{VO}_{4}\right)$, $10 \mathrm{mM}$ Sodium Fluoride, $10 \mathrm{ug} / \mathrm{ml}$ Leupeptin, $10 \mathrm{ug} / \mathrm{ml}$ Antipain, 100 units $/ \mathrm{ml}$ Aprotinin. After 20 strokes in a Dounce homogenizer, aliquots of total extracts were stored at $-80^{\circ} \mathrm{C}$ until use and the remaining homogenated samples were gently extracted $30 \mathrm{~min}$ on ice, and then centrifugated at $35.000 \times \mathrm{g}$ for $35 \mathrm{~min}$ at $4^{\circ} \mathrm{C}$ in Ultracentrifuge (Beckman-OPTIMA ${ }^{\mathrm{TM}}$ ). The supernatant (cytosolic fractions) was precipitated with 8 volumes of Acetone while the pellet (membrane fraction) extracted in Buffer A containing $0,1 \%$ Triton X 100 as described above. After centrifugation at $5.000 \mathrm{rpm}$ in Sorvall-SW41 rotor, the cytosolic and membrane protein pellets were resuspended in buffer containing $2 \%$ SDS with protease and phosphatase inhibitors. An aliquot of total lysates, cytosolic and membrane fractions were used to evaluate the amount of proteins [32].

Equal amounts of sperm's total, cytosolic and membrane proteins (50-100 $\mu \mathrm{g} /$ lane) were separated by $8 \%-10 \%$ SDS-PAGE and then transferred onto nitrocellulose (Hybon C Extra, Amersham Bioscience) following standard procedures. Brain proteins were used as positive control The membrane was blocked for 1 hour at room temperature with Tris Buffer Saline (TBS: $20 \mathrm{mM}$ Tris $\mathrm{pH}$ 7.6, $150 \mathrm{mM}$ ) plus $3 \%(\mathrm{w} / \mathrm{v})$ non fat dry milk and immunoblottings were performed with the following antibodies: 1:200 dilution of anti- PLC- $\gamma 1$ (Santa Cruz), 1:4000 dilution of anti-Phosphotyrosine antiboby clone 4G10 (UPSTATE) or anti-alpha Tubulin antiboby clone B-5-1-2 (SIGMA) for 2 hours at room temperature in TBS containing $1 \%(\mathrm{w} / \mathrm{v})$ BSA and $0.05 \%(\mathrm{v} / \mathrm{v})$ Tween 20 . After incubation, the membrane was washed four times for 5 min. with TBS containing $0.05 \%(v / v)$ Tween 20 and then incubated for 1 hour at room temperature with 1:4000 dilution of goat anti mouse or anti-rabbit IgG horseradish peroxidase (HRP) conjugated (Santa Cruz) in TBS containing $1 \%(\mathrm{w} / \mathrm{v})$ non fat dry milk and $0.05 \%(\mathrm{v} / \mathrm{v})$ Tween 20. The signal was developed by ECL detection system (Amersham Bioscience). When necessary, the nitrocellulose membranes were stripped with the following 
buffer (62,5 mM Tris HCL pH 6.8, 2\% (w/v) SDS, $100 \mathrm{mM}$ 2-mercapto-ethanol for $45 \mathrm{~min}$ at $60 \mathrm{C}^{\circ}$ with constant shacking. The membranes were then extensively PBS washed and then reprobed as described.

\section{Sperm pre-embedding electron microscopic immunocytochemistry}

Phospholipase C $\gamma 1$ (PLC- $\gamma 1$ ) localization was performed on $\mathrm{T} 0$, and on spermatozoa incubated under capacitating conditions with or without CD (CTR and CD samples). All different groups of spermatozoa were collected and immediately fixed in $2 \%$ paraformaldehyde and $0.5 \%$ glutaraldehyde in $0,1 \mathrm{M}$ cacodylate buffer $\mathrm{PH} 7.4$ for $1 \mathrm{~h}$ at $4^{\circ} \mathrm{C}$. After washing spermatozoa in PBS/1\% BSA/0.05\% Tween 20 by two centrifugations at $800 \mathrm{~g}$ for $5 \mathrm{~min}$., samples were resospended in Goat Serum (Sigma) 1:5 PBS/1\% BSA for $1 \mathrm{~h}$ at room temperature, and then incubated in a rabbit anti - PLC- $\gamma 1$ (Santa Cruz) 1:100 in PBS/1\% BSA overnight at $4^{\circ} \mathrm{C}$. The anti - PLC- $\gamma 1$ was then visualized with an anti-rabbit IgG - Gold colloidal Particle - $10 \mathrm{~nm}$ (EY Laboratories, Inc.) 1:20 in PBS/1\% BSA for $1 \mathrm{~h}$ at room temperature. After several washes in PBS/1\% BSA/ $0.05 \%$ Tween 20 spermatozoa were washed in $0.1 \mathrm{M}$ cacodylate buffer $\mathrm{PH} 7.4$ fixed with $2 \%$ paraformaldehyde and $0.5 \%$ glutaraldehyde in $0.1 \mathrm{M}$ cacodylate buffer $\mathrm{PH} 7.4$ for $1 \mathrm{~h}$ at $4^{\circ} \mathrm{C}$ and postfixed with $1 \%$ osmium tetroxide in $0.1 \mathrm{M}$ cacodylate buffer $\mathrm{PH} 7.4$ for $30 \mathrm{~min}$. at $4^{\circ} \mathrm{C}$. The samples were then dehydrated with a graded series of ethanol and embedded in LR White resin (Polyscience, Inc.). Ultrathin sections were stained with uranyl acetate and lead citrate before being examined in a Zeiss transmission electron microscope (EM900).

\section{ZP-induced intracellular calcium rise}

The calcium probe fluo-3-AM was used to assess the variations in the intracellular calcium concentration in response to the sZP coincubation. To this aim a $2 \mathrm{mM}$ stock solution of fluo-3-AM was solubilized in dry dimethylsulphoxyde, containing 37.5 g/l Pluronic F-127. Spermatozoa $\left(5 \times 10^{6}\right.$ spermatozoa $/ \mathrm{ml}$ final concentration) were incubated at $38.5^{\circ} \mathrm{C}$ for $30 \mathrm{~min}$ in Dulbecco's phosphate buffer with $\mathrm{Ca}^{2+}$ and $\mathrm{Mg}^{2+}$ containing $4 \mu \mathrm{M}$ fluo-3-AM avoiding the exposition of samples to the light. The samples were, then, putted in a warmed chamber containing the spermatozoa suspended in Dulbecco's phosphate buffer with $\mathrm{Ca}^{2+}$ and $\mathrm{Mg}^{2+}$ and observed under confocal microscopy (Radiance 2000, BioRad, UK) for $3-4 \mathrm{~min}$. The first $60-100 \mathrm{sec}$. of examination were carried out to exclude photobleaching. When the sample fluorescence emission was stable, an aliquot of sZP was added and the observation was performed for 3-5 min. To confirm the responsiveness of the system, the not responding samples were exposed to $10 \mu \mathrm{M}$ A23187 to assess their ability to respond to the calcium ionophore-induced entry. Only responding spermatozoa were considered as functionally integer. All the samples were assessed using the same exposition parameters to avoid artefacts in the data analysis. The fluorescence emission, expressed as Fluorescence Arbitrary Units, was proportional to the intracellular calcium concentration.

\section{Image analysis}

The acquisition of images by confocal microscopy was carried out using Lasersharp 2000 software (Biorad, UK). The image analysis was realized by LaserPix 4.0 (Biorad, UK).

\section{Statistical analysis}

Data reported in this paper are expressed as mean \pm standard deviation of three independent experiments, each performed in duplicate. The data were checked for normal distribution (Shapiro-Wilks W test) and then compared by ANOVA test (StatistiKL Version $\beta$ ). The differences were considered significant and highly significant for $\mathrm{p}$ values of $<0.05$ and $<0.01$, respectively.

\section{Acknowledgements}

The Authors would like to thank Dr. Oriana Di Giacinto and Dr. Delia Nardinocchi for their technical assistance. The Authors would like to thank the anonymous reviewers for their valuable comments and suggestions to improve the quality of the paper. This research was supported by PRIN2006 to $\mathrm{MM}$ and $\mathrm{BB}$.

\section{Authors' contributions}

NB have made substantial contributions to conception and design of the work and carried out the data analysis; AM performed the protein electrophoresis experiments; VR performed the TEM experiments; PL carried out the in vitro experiments; PB, MM and BB participated to the experimental design and have been involved in revising the manuscript critically for important intellectual content. All Authors read and approved the final manuscript.

Received: 10 August 2010 Accepted: 30 March 2011 Published: 30 March 2011

\section{References}

1. Knoll AH, Javaux EJ, Hewitt D, Cohen P: Eukaryotic organisms in Proterozoic oceans. Phil Trans R Soc B 2006, 361:1023-1038.

2. Weng G, Bhalla US, lyengar R: Complexity in biological signaling systems. Science 1999, 284:92-95.

3. Barabási AL, Oltvai ZN: Network biology: understanding the cell's functional organization. Nature Reviews 2004, 5:101-113.

4. Albert : Scale-free networks in cell biology. J Cell Sci 2005, 118:4947-4957.

5. Bernabò N, Mattioli M, Barboni B: The spermatozoa caught in the net: the biological networks to study the male gametes post-ejaculatory life. BMC Syst Biol 2010, 4:87.

6. Gur Y, Breitbart H: Mammalian sperm translate nuclear-encoded proteins by mitochondrial-type ribosomes. Genes Dev 2006, 20:411-416.

7. Mattioli M, Barboni B, Lucidi P, Seren E: Identification of capacitation in boar spermatozoa by chlortetracycline staining. Theriogenology 1996, 45:373-381.

8. Barabási AL, Albert R: Emergence of scaling in random networks. Science 1999, 286:509-512.

9. Breitbart H, Cohen G, Rubinstein S: Role of actin cytoskeleton in mammalian sperm capacitation and the acrosome reaction. Reproduction 2005, 129:263-268. 
10. Storey BT: Mammalian sperm metabolism: oxygen and sugar, friend and foe. Int J Dev Biol 2008, 52:427-437.

11. Dominguez R: Actin filament nucleation and elongation factorsstructure-function relationships. Crit Rev Biochem Mol Biol 2009, 44:351-66.

12. Campellone KG, Welch MD: A nucleator arms race: cellular control of actin assembly. Nat Rev Mol Cell Biol 2010, 11:237-51.

13. Carlsson AE: Actin dynamics: from nanoscale to microscale. Annu Rev Biophys 2010, 39:91-110.

14. Visconti PE, Bailey JL, Moore GD, Pan D, Olds-Clarke P, Kopf GS: Capacitation of mouse spermatozoa. I. Correlation between the capacitation state and protein tyrosine phosphorylation. Development 1995, 121:1129-1137.

15. Urner F, Sakkas D: Protein phosphorylation in mammalian spermatozoa. Reproduction 2003, 125:17-26.

16. Barbonetti A, Vassallo MR, Cinque B, Antonangelo C, Sciarretta F, Santucci R, D'Angeli A, Francavilla S, Francavilla F: Dynamics of the global tyrosine phosphorylation during capacitation and acquisition of the ability to fuse with oocytes in human spermatozoa. Biol Reprod 2008, 79:649-656.

17. Bravo MM, Aparicio IM, Garcia-Herreros M, Gil MC, Peña FJ, Garcia-Marin LJ: Changes in tyrosine phosphorylation associated with true capacitation and capacitation-like state in boar spermatozoa. Mol Reprod Dev 2005, 71:88-96.

18. Spungin $\mathrm{B}$, Breitbart $\mathrm{H}$ : Calcium mobilization and influx during sperm exocytosis. J Cell Sci 1996, 109:1947-1955.

19. Breitbart H: Signaling pathways in sperm capacitation and acrosome reaction. Cell Mol Biol 2003, 49:321-327.

20. Tulsiani DR, Zeng HT, Abou-Haila A: Biology of sperm capacitation: evidence for multiple signalling pathways. Soc Reprod Fertil Supp/ 2007, 63:257-272

21. Abou-haila A, Tulsiani DR: Signal transduction pathways that regulate sperm capacitation and the acrosome reaction. Arch Biochem Biophys 2009, 485:72-81.

22. Oikonomopoulou I, Patel H, Watson PF, Chantler PD: Relocation of myosin and actin, kinesin and tubulin in the acrosome reaction of bovine spermatozoa. Reprod Fertil Dev 2009, 21:364-377.

23. Correa $L M$, Thomas A, Meyers SA: The macaque sperm actin cytoskeleton reorganizes in response to osmotic stress and contributes to morphological defects and decreased motility. Biol Reprod 2007, 77:942-953.

24. Cohen $G$, Rubinstein $S$, Gur Y, Breitbart H: Crosstalk between protein kinase $\mathrm{A}$ and $\mathrm{C}$ regulates phospholipase $\mathrm{D}$ and $\mathrm{F}$-actin formation during sperm capacitation. Dev Biol 2004, 267:230-41.

25. Janmey PA: The cytoskeleton and cell signaling: component localization and mechanical coupling. Physiol Rev 1998, 78:763-778.

26. Shafrir $Y$, ben-Avraham D, Forgacs $G$ : Trafficking and signaling through the cytoskeleton: a specific mechanism. J Cell Sci 2000, 113:2747-2757.

27. Forgacs $G$, Yook SH, Janmey PA, Jeong H, Burd CG: Role of the cytoskeleton in signaling networks. J Cell Sci 2004, 117:2769-2775.

28. Bernabò N, Tettamanti E, Pistilli MG, Nardinocchi D, Berardinelli $P$, Mattioli $\mathrm{M}$, Barboni B: Effects of $50 \mathrm{~Hz}$ extremely low frequency magnetic field on the morphology and function of boar spermatozoa capacitated in vitro. Theriogenology 2007, 67:801-815.

29. Bernabò N, Tettamanti E, Russo V, Martelli A, Turriani M, Mattioli M, Barboni B: Extremely low frequency electromagnetic field exposure affects fertilization outcome in swine animal model. Theriogenology 2010, 73:1293-1305

30. Bernabò N, Pistilli MG, Mattioli M, Barboni B: Role of TRPV1 channels in boar spermatozoa acquisition of fertilizing ability. Mol Cell Endocrinol 2010, 323:224-231

31. Barboni $B$, Mattioli $M$, Seren E: Influence of progesterone on boar sperm capacitation. J Endocrinol 1995, 144:1-8.

32. Lowry OH, Rosebrough NJ, Farr AL, Randall RJ: Protein measurement with the Folin phenol reagent. J Biol Chem 1951, 193:265-275.

doi:10.1186/1752-0509-5-47

Cite this article as: Bernabò et al.: The role of actin in capacitationrelated signaling: an in silico and in vitro study. BMC Systems Biology 2011 5:47.

\section{Submit your next manuscript to BioMed Central and take full advantage of:}

- Convenient online submission

- Thorough peer review

- No space constraints or color figure charges

- Immediate publication on acceptance

- Inclusion in PubMed, CAS, Scopus and Google Scholar

- Research which is freely available for redistribution 